\title{
Enabling Scalable Chiplet-based Uniform Memory Architectures with Silicon Photonics
}

\author{
Pouya Fotouhi* \\ pfotouhi@ucdavis.edu \\ Department of Electrical and Computer Engineering \\ University of California \\ Davis, California \\ Jason Lowe-Power \\ jlowepower@ucdavis.edu \\ Department of Computer Science \\ University of California \\ Davis, California
}

\author{
Sebastian Werner ${ }^{*}$ \\ swerner@ucdavis.edu \\ Department of Electrical and Computer Engineering \\ University of California \\ Davis, California \\ S.J. Ben Yoo \\ sbyoo@ucdavis.edu \\ Department of Electrical and Computer Engineering \\ University of California \\ Davis, California
}

\begin{abstract}
Chiplet-based systems have recently received much attention for scaling-up processing power in HPC systems due to their high energy efficiency and low cost manufacturing; however, large interchiplet NUMA latencies, distance-related energy overheads, and limited IO bandwidth caused by state-of-the-art packaging and interconnect technologies substantially limit their scalability. Large last level caches (up to $16 \mathrm{MiB} /$ chiplet and $40 \%$ of chiplet area) of current systems can only temporarily hide these limitations and come at the large cost and leakage power of SRAM cells. In this paper, we propose the use of integrated silicon-photonic ( $\mathrm{SiPh})$ interconnects on an organic package substrate which combines low material costs with a high IO bandwidth, distance-independent energy consumption, and low-latency point-to-point interconnection fabric to effectively overcome current interconnect and packaging limitations. We exploit the properties of this fabric to propose a scalable uniform memory architecture (S-UMA) that overcomes all NUMA-related performance challenges. Moreover, we propose exploiting our low-latency SiPh fabric to remove the large LLC caches from the processor chiplets and re-integrate them into separate chiplets, increasing manufacturing yield by using smaller chiplets, allowing to use the most efficient process for SRAM circuits, or easing integration of alternative memory technologies without performance hits. Compared to state-of-the-art architectures, S-UMA offers $23 \%$ performance speed-up and $30 \%$ network power savings on average across HPC workloads for a 8-chiplet 64-core system.
\end{abstract}

${ }^{*}$ Both authors contributed equally to this work.

Permission to make digital or hard copies of all or part of this work for personal or classroom use is granted without fee provided that copies are not made or distributed for profit or commercial advantage and that copies bear this notice and the full citation on the first page. Copyrights for components of this work owned by others than ACM must be honored. Abstracting with credit is permitted. To copy otherwise, or republish, to post on servers or to redistribute to lists, requires prior specific permission and/or a fee. Request permissions from permissions@acm.org.

MEMSYS '19, September 30-October 3, 2019, Washington, DC, USA

(C) 2019 Association for Computing Machinery.

ACM ISBN 978-1-4503-7206-0/19/09..\$15.00

https://doi.org/10.1145/3357526.3357564

\section{CCS CONCEPTS}

- Computer systems organization $\rightarrow$ Interconnection architectures; • Hardware $\longrightarrow$ Photonic and optical interconnect.

\section{KEYWORDS}

Chiplets, Interconnects, Memory Architecture, Silicon Photonics

\section{INTRODUCTION}

Growing data sets in modern workloads are driving the need for higher processing power and memory bandwidth/capacity in HPC systems. Unfortunately, the slowing down of Moore's law reduces the ability to attain higher processing power and memory capacity within a single compute node ("scale-up") for each generation of silicon technology, forcing system designers to add compute nodes ("scale-out") to satisfy performance demands. Scaling-out, however, leads to distributed memory architectures with compute nodes operating in different address spaces and explicitly communicating and managing coherence through software (e.g. message passing), thereby causing significant performance and energy overheads for data movement between compute nodes-a key challenge in current HPC systems. Scaling-up shared memory architectures within a single address space, on the other hand, allows hardware-managed coherence which is significantly faster and allows programmers to focus on what matters for parallel speed-ups rather than communication and synchronization. Moreover, the cost of providing hardware coherence (i.e., traffic, storage cost for tracking sharers, latency, and energy) is generally considered to scale gracefully with the core count for hierarchies in modern systems [42].

One significant bottleneck to further scaling-up processing power within tight power envelopes are the growing monolithic silicon development and manufacturing costs which have seen a $7 \times$ increase from $28 \mathrm{~nm}$ to $7 \mathrm{~nm}$ and have lead companies to increasingly rely on breaking monolithic chips into smaller "chiplets" [15]. Utilizing several smaller chiplets assembled using advanced packaging technologies instead of one large monolithic chip reduces costs by exploiting the higher yield of smaller dies at low performance and energy overheads through tight integration in the same package [34]. In addition, it allows freedom of mixing and matching the most cost- and power-efficient process nodes for chiplets, particular for those with harder-to-shrink or purpose-built components, 
thereby representing a highly promising technique to support the trend of increasingly heterogeneous computing systems.

Looking ahead, we can expect future systems to further exploit the cost benefits of chiplets to scale-up performance at acceptable power and cost by increasing the total number of chiplets within a single package-a trend observed in several recent commercial designs $[1,3,55]$; however, to enable further scaling of chiplet-based systems, several key challenges must be addressed:

1. Interconnection Challenge. The dependency of energy consumption on interconnect length coupled with centimeter-scale chiplet sizes only allows interconnecting adjacent chiplets (on a planar layout) without excessive crosstalk and energy overheads. This leads to low-radix/high-diameter topologies with high average hop counts in which each inter-chiplet hop imposes tens of nanoseconds latency [71]. Given these latency overheads, inter-chiplet communication in general, but cache coherence traffic (often requiring multi-hop coherence protocols) in particular, can now significantly degrade system performance and thereby limit scalability.

2. NUMA Challenge. Current designs with low numbers of chiplets are already non-uniform memory architectures (NUMAs) exhibiting significant variances in access latency to different addresses [71]. NUMA systems are notoriously difficult to program, making it extremely challenging for programmers to extract performance. Energy and performance limitations on the interconnect exacerbates this issue by making scalable chiplet-based UMAs difficult to attain with acceptable energy efficiency. Recent solutions aiming to make chiplet-based systems more UMA by implementing a central IO chip through which all traffic passes through are temporary remedies [55], but will be performance and power bottlenecked by the switching fabric on the IO die.

3. Disintegration Limits. Not only does the interconnect challenge limit scalability of chiplet-based systems, but also how much processor disintegration-the process of breaking one large manycore processor into smaller ones-can be exploited. As a result, designers will be forced to opt for few large rather than many small chiplets due to unacceptable latency overheads on the interconnect, despite it incurring much more manufacturing costs and limiting the freedom of choosing the most suitable process node.

4. Packaging Challenge. Accommodating larger numbers of chiplets in the same package requires larger substrates. Silicon interposers offer high IO density but are too expensive for the system sizes in current HPC nodes which mainly use cheaper organic substrate with lower IO density [46]. However, IO density is crucial to satisfy future bandwidth demands of inter-chiplet links. Silicon bridges [30] integrated into organic substrates connect the edges of tightly-coupled chiplets with very high IO density, but can only connect physically-adjacent chiplets. Clearly, interconnects with high IO density and energy-efficient signaling over long distances integrated into a cost-efficient organic package substrate are in high demand.

Integrated optical links enabled by Silicon Photonics (SiPhs) provide low latency, high bandwidth density through wavelengthdivision multiplexing (WDM), and distance-independent energy consumption, and can now be integrated on organic package substrates [46], making them an attractive technology for inter-chiplet connectivity. Moreover, SiPh devices can exploit WDM to perform wavelength-selective routing which allows a chiplet to connect to multiple other chiplets through a single optical IO pin (addressing them on different wavelengths), enabling high-radix low-diameter networks. Optical networks provide sufficient scalability in terms of crosstalk and power consumption to enable point-to-point connectivity between up to 32 nodes with bisection bandwidths matching current chiplet-based systems [67]-a design option infeasible with electrical interconnects due to large amounts of wiring, distancedependent energy consumption, and IO pin requirements.

In this paper, we propose a novel chiplet-based scalable UMA (SUMA) exploiting $\mathrm{SiPh}$ interconnects to solve all above mentioned challenges. We make the following architectural contributions:

- A scalable and compact $\mathrm{SiPh}$ point-to-point interconnection fabric integrated on an organic package enabling a chiplet-based uniform memory architecture with distanceindependent energy consumption and latency and low pin IO requirements by exploiting WDM and wavelength-selective routing.

- Dis- and re-integration of large LLCs from processor to separate chiplets containing LLCs, directory and memory controllers to enable lower leakage power of optimized processes for SRAM, cost reductions through higher manufacturing yield of smaller chiplets, and more flexibility in implementing heterogeneous memory technologies with less integration complexity. The high-bandwidth SiPh point-to-point interconnection between processor and LLC chiplets amortize any off-chiplet LLC access latency overheads.

- Integration of SiPh transceivers (TRXs) fabricated on separate dies rather than monolithically integrated on the chiplets to enable the freedom of choosing the most appropriate processes, prevent the reduced yield of $\mathrm{SiPh}$ components to decrease the yield of processor chiplets, and to remove potential area concerns of $\mu \mathrm{m}$-scale SiPh components. Silicon bridges tightly connect TRXs and chiplets with very high IO density at energy and latency similar to on-chip wires, causing negligible overheads.

While a few previous works have studied SiPh for chip-to-chip communication on-board [18, 36] or interposers [67], this paper is, to the best of our knowledge, the first to evaluate the energy and performance benefits of a point-to-point SiPh interconnect and its ability to provide a scalable chiplet-based UMA system integrated on an organic package substrate. Our evaluation results show that S-UMA provides a speed-up of $23 \%$ and reduces network power consumption by $30 \%$ compared to state-of-the-art chipletbased NUMA and provide a cost-efficient, practical way for scalable chiplet-based UMA systems. Therefore, we think that S-UMA could be a key enabler with long-term impact for the future of scaling-up processing power of HPC nodes that exploit the benefits of chipletbased systems: high energy efficiency through tight, heterogeneous integration and reduced cost through processor disintegration.

\section{CHIPLET-BASED SYSTEMS: CHALLENGES AND OPPORTUNITIES}

Chiplet-based systems integrate and interconnect several-possibly heterogeneous-processor and/or memory dies in the same package. Figure 1 illustrates an example layout of such a system with CPUs, GPUs, FPGAs, and an IO die for connecting chiplets with each other 


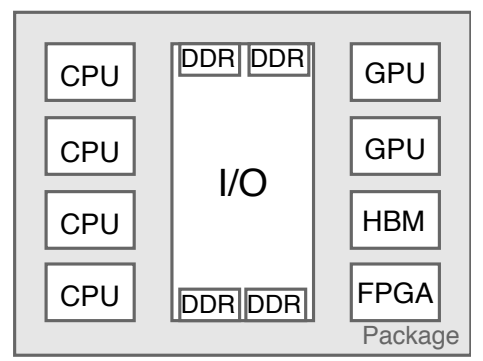

Figure 1: An example chiplet-based system with heterogeneous processor dies integrated in the same package

and to DDR memory interfaces. In the following, we will review and analyze state-of-the-art packaging and integration technologies as they are a key design factor with significant implications on manufacturing cost, interconnect density and energy, and thereby on the scalability and future outlook of chiplet-based systems.

\subsection{Packaging and Interconnect Technologies}

Various chiplet-based system architectures from different vendors have emerged in recent years based on different packaging technologies, each with different benefits and trade-offs regarding cost, performance, energy, and scalability, as well as implications on the memory subsystem, interconnection network, and overall system design. Figure 2 illustrates the state-of-the-art techniques deployed in current commercial designs: 1) Multi-Chip Modules (MCMs) [1, $22,29,44,55]$; 2) 2.5D integration with a silicon interposer [3335, 40, 41, 45, 72]; and 3) Silicon Bridges (like in Intel's Embedded Multi-Die Interconnect Bridge (EMIB) technology) [30,56]. Table 1 provides a summary and comparison of each integration technique, which we will discuss in the following.

2.1.1 Multi-Chip Modules (MCMs). MCMs (Figure 2a) mount and connect chiplets with high-density interconnects (HDIs) on the package substrate using wire-bond or flip-chip technology [46]. MCMs typically utilize organic package substrates as these are not manufactured in the foundry (as opposed to Silicon interposers) and therefore much cheaper. In addition, no further processing steps are needed (e.g., 2.5D integration needs additional processing step for the vertical interconnects), making MCMs the cheapest option from both a material and processing cost perspective, and especially attractive for systems of larger scale. For instance, packages of
MCMs deployed in current HPC nodes can be around $10 \mathrm{~cm} \times 10 \mathrm{~cm}$ in size $[12,29,55])$.

Challenges. Wire-bond or flip-chip interconnects offer relatively low IO pin densities, thereby restricting off-chip(let) bandwidth. High IO density, however, is crucial as chips are facing a "pin wall" where the vast majority of pins is dedicated to power/ground, leaving very few pins to satisfy off-chip communication demands [58]. Although current electrical interconnect technologies on organic substrates $(20 \mathrm{~Gb} / \mathrm{s}$ operation per IO pin at $0.54 \mathrm{pJ} / \mathrm{bit}$ over $4.5 \mathrm{~mm}$ at $28 \mathrm{~nm}$ [50]) appear to satisfy the bandwidth demands of current systems [1], higher pin data rates are difficult to attain due to excessive crosstalk of electrical signaling.

In addition, high IO pitches can also restrict the number of chiplets a chiplet can be connected to. From a network perspective, this leads to low-radix chiplets requiring networks with high diameters and average hop counts. Inter-chiplet hop latencies have a large impact on system performance (more than 30ns per hop in current systems $[1,71]$ ) and lead to complex NUMA systems with high latencies variations. Topologies can exhibit lower diameters with the same radix by connecting distant nodes; however, energy grows linearly with distance for electrical links, making this approach infeasible for the dimensions in chiplet-based systems.

Therefore, although attractive from a cost perspective, the bandwidth and distance related challenges of MCMs severely limit their ability to satisfy the performance demands of future chiplet-based systems of larger scale.

2.1.2 2.5D Integration with Silicon(Si) Interposers. 2.5D integration (Figure $2 b$ ) places an additional silicon die on top of the package substrate, and the chiplets on top of the interposer. Chiplets connect to each other and to the package substrate through the interposer with through-silicon vias (TSVs) and $\mu$ bumps. Interposers can be passive (interconnects only) or active (interconnects and logic) [59]. The main benefit of $2.5 \mathrm{D}$ integration is the substantially higher interconnection density compared to MCMs [4, 52, 64], either allowing for higher maximum bandwidth or for lower energy per bit by reducing the data rates of the IO transceivers.

Challenges. 2.5D integration with $\mathrm{Si}$ interposers overcomes the challenges of MCMs by offering higher IO pin density through smaller pitches of $\mu$ bumps and TSVs $[6,16,53]$; however, $\mathrm{Si}$ is significantly more expensive than organic substrates and $2.5 \mathrm{D}$ integration requires additional (and more complex) processing steps. Material costs could be somewhat amortized through high-volume manufacturing, but the very-large size needed for large-scale chiplet-based

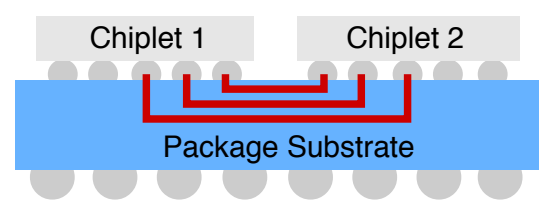

(a) Multi-Chip Module

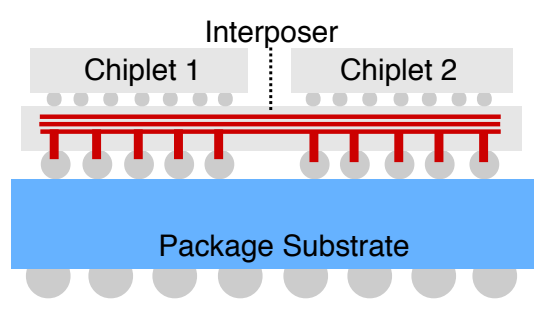

(b) Silicon Interposer

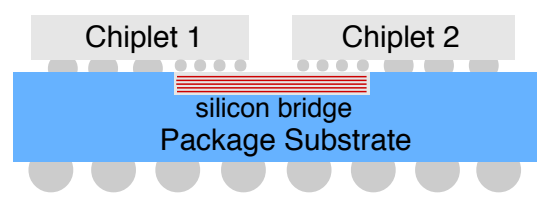

(c) Silicon Bridge

Figure 2: State-of-the-art Integration Technologies for Chiplet-to-chiplet Interconnection 
Table 1: Summary and properties of state-of-the-art integration techniques. Note that these values vary depending on the technology node, interconnect length, and optimized integration approaches; however, general trends and physical limitations stated in this table hold true nevertheless.

\begin{tabular}{ccccc}
\hline & $\mathrm{MCM}$ & 2.5D Integration & Silicon Bridge & SiPh MCM \\
\hline Materials & Organic substrate & Si interposer & Organic substrate, Si bridge & Organic substrate, Polymer \\
Material Cost & $\$$ & $\$ \$ \$$ & $\$ \$$ & $\$$ \\
Pin BW & $20 \mathrm{Gbps}$ & $28 \mathrm{Gbps}$ & $28 \mathrm{Gbps}$ & $160-640 \mathrm{Gbps}^{3}$ \\
Pin Pitch & & $6 \mu \mathrm{m}$ & $2 \mu \mathrm{m}$ & $<\mu \mathrm{m}$ \\
$\mathrm{pJ} / \mathrm{bit}^{4} \mathrm{Gbps}^{5}$ & $0.027(4.5 \mathrm{~mm})$ & $0.0114(3.5 \mathrm{~mm})$ & $<0.035(1 \mathrm{~mm})$ & $0.017(\mathrm{several} \mathrm{cm})$ \\
\hline
\end{tabular}

systems in the HPC domain would still make Si economically unreasonable.

Although solving the IO density issues of MCMs, 2.5D integration cannot overcome the limitations imposed by interconnect length. High IO density can enable higher-radix switches on the chiplets (i.e., connect each chiplet to more other chiplets), thereby reducing network diameter; however, chiplets are relatively large $\left(\sim 1 \mathrm{~cm}^{2}\right)$ and are laid out on 2D planar floorplan, meaning that connecting to chiplets that are not directly adjacent requires to route electrical interconnects over $\mathrm{cm}$-scale distances, which will require repeater and buffer circuitry that lead to very high energy per bit, especially for high-speed links. Acceptable energy can thus only be provided on links connecting to adjacent chiplets, thereby also limiting the radix and its impact on the network diameter.

2.1.3 Silicon bridges. Si bridges, like Intel's EMIB technology (Figure 2c), aim to solve the limitations of both MCM (poor interconnection density) and 2.5D integration (high cost for Si interposer) by embedding small and thin (less than 75 $\mu m$ [30]) Si chips ("bridges") with (currently) four metal layers into an organic package substrate to interconnect the edges of adjacent chiplets. Si bridges offer very high IO density with latency and energy metrics similar to on-chip wires (by integrating fine-pitched "back end of line" (BEOL) interconnects) and enable short interconnects through tight packaging with just $100 \mu \mathrm{m}$ between chiplets. Si bridges thereby offer a more scalable solution by combining the low material costs of organic substrates with the high IO bandwidth density of Si interposers.

Challenges. Just like Si interposers and MCMs, Si bridges utilize electrical interconnects and thus impose the same distance-related energy limitations, and thereby the same network radix/diameter problem. Consequently, Si bridges alone cannot overcome the NUMA, interconnect, and scalability challenges in chiplet-based computing systems. Systems with one large chiplet and several (much) smaller chiplets could exploit the high IO density of Si bridges to directly connect the large chiplet to each small chiplet, but inter-chiplet traffic would likely be bottlenecked by the crossbar on the large chiplet, limiting the scalability of this approach. In fact, the vast majority of current designs utilize the batch processing and design re-use benefits processor disintegration (integrating smaller, replicated processor chips) $[1,29,34,41,72]$, benefits that could be exploited even more in systems of larger scale.

\subsection{Packaging: Implications on System Design}

In summary, although each integration technology comes with its own benefits and trade-offs (as listed in Table 1), the following fundamental challenges limiting all of these state-of-the-art integration technologies from supporting the trend towards larger systems that further exploit the benefits of chiplet-based systems remain:

Interconnect Restrictions. Current integration technologies can enable sufficient IO bandwidth density but are distance-limited due to the energy consumption of electrical signaling and large chiplet dimensions which prevents direct connectivity to chiplets other than direct neighbors on the substrate. Therefore, future systems are restricted to topologies with relatively high average hop counts, with each hop incurring tens of nanoseconds in latencysignificantly degrading system performance.

NUMA Challenge. The implications of the interconnect challenge on the average hop count and latency will cause substantially larger variances in memory access latency compared to current systems, which are already NUMA [71]. Extracting performance from NUMA systems is notoriously difficult for programmers, leaving much of the potential of the compute resources untapped. Moving closer to UMA (rather than in the opposite direction) is therefore critical, but is hard to attain in chiplet-based systems. Recent efforts making all chiplets communicate through an IO chip go in the right direction [55], but the limited scalability of a central interconnection fabric makes this only a temporary solution.

Scalability and Disintegration Limits. The limitations of electrical signaling over longer distances is one of the main reasons for the interconnect challenge and limits the scalability of future chiplet-based systems in which the large communication overheads may not be acceptable. The only option will then be to use fewer large chiplets to reduce off-chiplet communication and network diameter (and, in turn, latency); however, this removes one of the main motivators for chiplet-based systems, namely the lower cost of the higher manufacturing yield of smaller chiplets and the freedom of choosing the most efficient process node. A scalable, low-latency interconnection fabric is therefore key to future cost reductions.

Packaging Challenges. The size of high-end chiplet-based systems is already large and will further increase in the future, making

\footnotetext{
${ }^{1}$ For the current state of SiPhs integration, mainly due to higher engineering cost, lower volume, yield, and expensive tooling

${ }^{2}$ Based on maximum reported pin data rates $[1,27,50]$

${ }^{3}$ Based on 40Gbps PAM-4 transceivers and 4-16 wavelengths per link (more wavelengths are feasible but might lead excessive crosstalk and impractical laser costs) [5, 69]

${ }^{4}$ Based on minimum reported $\mu$ Bump pitches [6, 30, 31]

${ }^{5}$ Based on recently reported and utilized interconnects [17, 43, 50, 56]
} 
a inexpensive substrate material increasingly important and organic substrates the preferred solution. Those, however, can only provide sufficient IO bandwidth when combined with technologies like silicon bridges which are based on electrical signaling and thus distance-limited. Ideally, one would desire an interconnect technology on an organic substrate that overcomes the distance-related energy overheads of electrical interconnects, while providing high IO pin bandwidth.

\subsection{Opportunities with Silicon Photonics}

Integrated optical interconnects enabled by SiPhs offer properties that can be exploited to overcome all of the previous challenges of electrical interconnects. Optics offer virtually distance-independent energy consumption, near speed-of-light signal propagation latency, and high bandwidth density through wavelength-division multiplexing (WDM) which enables to transmits on multiple wavelengths in parallel inside the same optical link. Moreover, SiPh devices can perform wavelength-selective routing, i.e., data is routed based on the wavelength channel, which allows to connect a chiplet to multiple other chiplets through a single waveguide. In addition, SiPhs can be integrated on organic package substrates, which allows to overcome the interconnection challenges while enabling the use of a relatively cheap packaging substrate (compared to Si interposer). In particular, SiPhs can be used to solve the challenges of chiplet-based systems as follows:

Solving the Interconnect Challenge. WDM not only enables high IO pin bandwidth but also allows to communicate with several chiplets through the same pin (on different wavelengths). Moreover, energy consumption in now distance-independent and signaling fast enough to reach each chiplet on a package in less than a nanosecond (depending on the material, a few hundred $\mathrm{ps} / \mathrm{cm}[25,28,68])$. This solves any IO limitations in terms of communication bandwidth and enables low-latency low-diameter/highradix (and even point-to-point depending on scale) networks.

Solving the NUMA Challenge. A scalable low-latency lowdiameter network could allow large-scale chiplet-based systems to become UMA, thereby significantly facilitating programmability and, in turn, paving the way for easier and more efficient extraction of performance from the available computing resources.

Enabling Scalability and Further Disintegration. In addition to exploiting the latency, bandwidth density, and energy properties of $\mathrm{SiPh}$ interconnects and their integration on cheap organic substrates, their superior scalability (compared to electrical interconnects) can be used to further support disintegration of chiplets into even smaller chiplets. This not only improves manufacturing yield of chiplets, but also opens-up new opportunities for dis- and re-integration. For instance, large LLCs could be moved on separate chiplets and either be manufactured on a more efficient process for SRAM for lower leakage power or facilitate the integration of alternative memory technologies like non-volatile STT-RAM [37].

Enabling Low-cost Package Substrates. SiPh interconnects can be integrated on organic package substrate, thereby offering high pin IO bandwidth density without requiring expensive Si interposers or being restricted to short-distance communication.

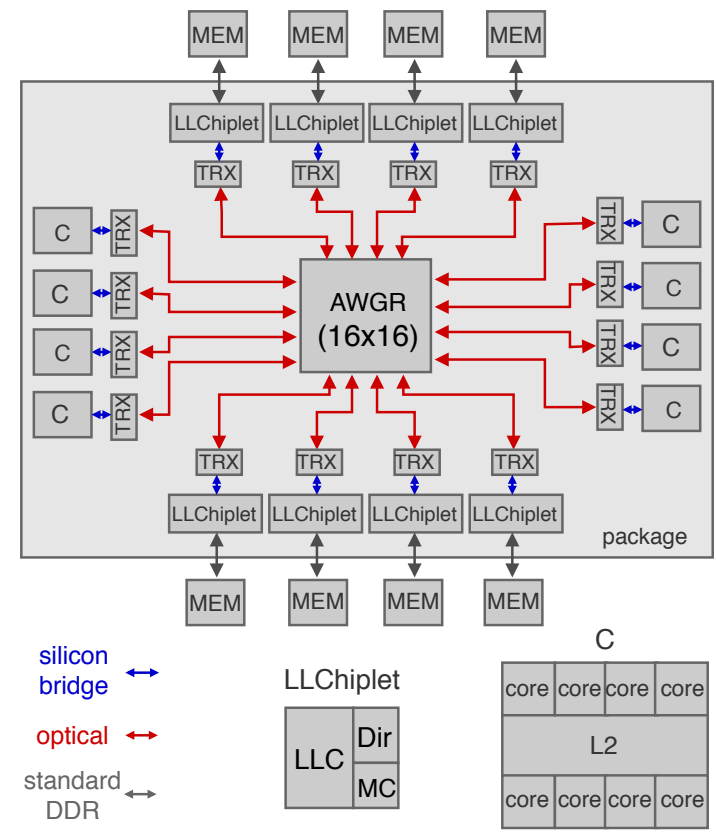

Figure 3: Target System (not to scale) with SiPh interconnection die (which is an AWGR point-to-point fabric), processor chiplets (C), LLChiplets (containing LLC, directory coherence controller (Dir) and memory controller (MC)), and SiPh transceiver chiplets (TRX).

We note, however, that $\mathrm{SiPh}$ come with their own challenges, one being manufacturing yield which is currently lower than for CMOS devices. Besides, the majority of SiPh chips are currently processed on older technology nodes (mostly $45 \mathrm{~nm} / 65 \mathrm{~nm}$ [24, 38, 62], 28nm has recently emerged [51]) leading to lower-volume fabrication compared to current $7 \mathrm{~nm}$ or $14 \mathrm{~nm}$ nodes for CMOS. This not only increases cost, but also makes it unreasonable to monolithically integrate chiplets with $\mathrm{SiPh}$ transceivers as the $\mathrm{SiPh}$ devices could render a correctly functioning chiplet defective. Moreover, thermal control of components has to be considered carefully to avoid malfunctioning of $\mathrm{SiPh}$ devices. In the following, we will discuss these issues in more detail and introduce practical solutions to largely overcome these challenges. In this context, we propose a system architecture that utilizes the properties of SiPhs to design a highperformance, energy-efficient, scalable chiplet-based UMA system on a cheap organic substrate.

\section{SCALABLE CHIPLET-BASED UNIFORM MEMORY ARCHITECTURE}

Figure 3 depicts a high-level view of our proposed system architecture, which we will discuss in detail in the following, consisting of many-core processor chiplets, LLChiplets containing last level caches (LLCs), memory and directory controllers, SiPh transceiver chiplets (TRX), and a SiPh all-to-all interconnection die in the same package. Our design incorporates several techniques to address the interconnect, NUMA, disintegration, and packaging challenges outlined in the previous sections and has the following goals: 


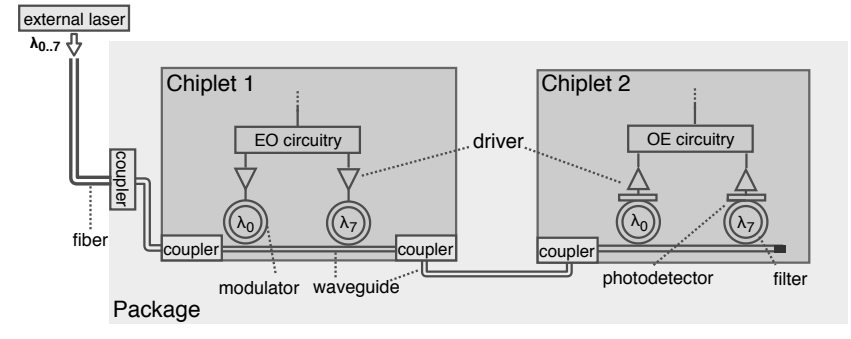

Figure 4: Example Inter-chiplet SiPh link. Modulators encode bits onto wavelength which are decoded by photodetectors at the receiver. Microring Resonators (MRRs) enable wavelength-selective modulation and detection.

(1) Utilize a scalable, low-latency, high-bandwidth, low-energy point-to-point $\mathrm{SiPh}$ interconnection fabric for inter-chiplet communication to enable a scalable chiplet-based uniform memory and cache architecture not attainable with electrical interconnects.

(2) Enable dis- and re-integration of LLC structures from processor into separate chiplets to reduce manufacturing cost and to provide more flexibility in choosing the most powerefficient process for the large LLCs.

(3) Offer a practical, cost-efficient chiplet-based system architecture with advanced $\mathrm{SiPh}$ packaging to support the trend of higher numbers of chiplets inside the same package.

This section introduces our proposed chiplet-based system, in particular the new techniques we propose to target the challenges of scalable chiplet-based systems.

\subsection{Addressing the Interconnect Challenge}

Electrical interconnects suffer from high distance-dependent energy and latency overheads on relatively long inter-chiplet links and offer limited IO pin bandwidth. Therefore, connecting chiplets to not physically-adjacent chiplets is prohibitive in terms of pin availability and energy, leading to multi-hop networks with larger latency overheads. Integrated optical interconnects enabled by $\mathrm{SiPh}$ overcome these challenges by offering almost distance-independent energy, high pin bandwidth density with WDM, and wavelengthselective routing which enables compact point-to-point switching fabrics with optical links growing linearly with the number of nodes (rather than quadratically as in the electrical domain).

3.1.1 SiPh Inter-Chiplet Links. Figure 4 illustrates the details of an inter-chiplet $\mathrm{SiPh}$ link on an organic substrate. An external laser generates light which is coupled from a fiber into waveguides integrated into the organic package substrate (fiber-to-package coupling) and from package waveguides into on-chiplet waveguides (package-to-chiplet coupling) and vice versa. ${ }^{1}$. Bits are encoded onto wavelength by modulators (one for each wavelength) which then traverse the waveguides and are filtered out and converted back into the electrical domain by photodetectors.

\footnotetext{
${ }^{1}$ For current technologies, an external lasers offer lower cost by providing higher manufacturing yield and independent testing. On-chip CMOS-compatible lasers have been demonstrated and would allow batch processing, but currently offer much lower laser efficiency, higher susceptibility to thermal fluctuations, and lower yield [63].
}

Microring resonators (MRRs) form the basis for both modulators and detectors and are designed to respond one particular resonance wavelength channel which must be controlled by MRR tuning circuitry to avoid resonance shifts due to ambient thermal fluctuations and manufacturing mismatches. Waveguides and MRRs typically have an area footprint less than $\sim 5 \mu \mathrm{m}$, while couplers are 200$400 \mu \mathrm{m}$ in length and $5-20 \mu \mathrm{m}$ in width $[5,13,14]$.

3.1.2 Arrayed Waveguide Grating Router. In order to support fast transfers of coherence and data traffic between chiplets, the interconnection fabric should ideally provide diameter-1 connectivity which could be enabled by a crossbar or a point-to-point network. WDM, aside from its bandwidth benefits, can be used to connect a single node to multiple receiver nodes by performing wavelength-selective routing within a single optical link. This allows implementing point-to-point networks without a large number of physically-interconnected port.

Several different SiPh devices have been explored and demonstrated to successfully enable a point-to-point switching fabric [5]. In this paper, we utilize the Arrayed Waveguide Grating Router (AWGR) [54] to provide point-to-point connectivity between all chiplets. Several previous studies have explored the use of AWGRs as the interconnection fabric in computing systems and show that AWGRs are a compact solution with lower loss, crosstalk, and thermo-optical control circuitry than alternative $\mathrm{SiPh}$ devices for point-to-point connectivity $[25,66,67]$. We note, however, that the choice of the $\mathrm{SiPh}$ fabric is orthogonal to our proposal which could be combined with other interconnect technologies offering similar point-to-point connectivity for providing UMA.

The AWGR is a passive SiPh switching platform providing pointto-point connectivity between each input and output port by using phase changes and constructive interference. Figure 5 a shows the wavelength routing properties of an AWGR. The wavelengths entering the waveguide on a given input port are evenly distributed to the output ports-one wavelength to one output port. Therefore, the number of wavelengths per input port must equal the number of output ports to provide all-to-all connectivity. For details on how wavelength distribution is achieved inside the AWGR on the technology level, we refer the reader to $[25,66]$. Figure $5 \mathrm{~b}$ shows a photograph of a manufactured $8 \times 8$ Silicon Nitride (SiN) AWGR which has a footprint of $\sim 1 \mathrm{~mm}^{2}$.

Figure 5a illustrates point-to-point connectivity by distributing one of the wavelengths of an input port to one output port each-the basic operation of a conventional AWGR. Routing WDM signals through an AWGR can also be provided either by (i) routing the WDM signals through a single bit-parallel AWGR [25, 26] or (ii) by using spatial-division multiplexing (SDM), i.e. integrating and transmitting data through several parallel AWGRs (either planar or 3D-stacked [60]). (i) requires the laser to output a wider wavelength spectrum and leads to higher crosstalk inside the AWGR and, in turn, high laser power. (ii) has significantly lower laser requirements but needs larger die area or more SiPh layers, as well as more optical IO pins. However, $\mathrm{SiPhs}$ generally require very few pins with relatively small pin pitches and inter-chiplet bandwidth can conveniently be attained with SiPh, e.g. matching AMD's 20GB/s IF bandwidth [71] requires eight wavelengths with highly energy-efficient $20 \mathrm{~Gb} / \mathrm{s}$ transceivers [39, 73]. Minimizing crosstalk and laser power would 


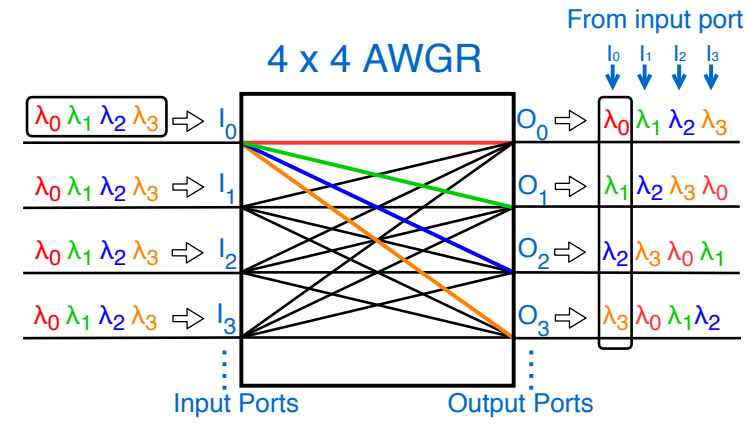

(a) Wavelength Switching Pattern

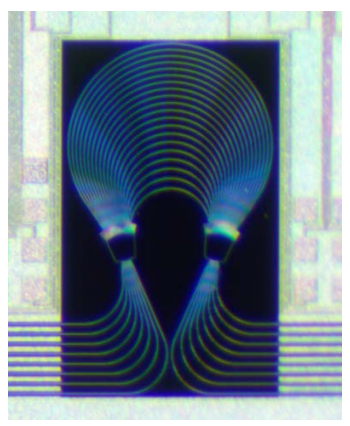

(b) Layout

Figure 5: AWGR: (a) Wavelength $(\lambda)$ switching mechanism and (b) physical layout of an $8 \times 8$ SiN AWGR [54])

thus require eight AWGR which could conveniently be integrated since an AWGR has a relatively small footprint (especially with 3Dstacking [60]) and is implemented on a separate die. We therefore apply the SDM approach in S-UMA to minimize system cost and laser power by reducing the number of wavelengths and lasers.

\subsection{Addressing the NUMA Challenge}

The overall performance in large-scale computing systems running workloads with ever-growing data sets extremely depends on the performance of the memory subsystem. In fact, to scale aggregate processing power, current system designs (e.g., Centaurs on IBM powers [57] or buffer chips in Oracle M series [47]) increasingly rely on NUMAs implementing ever-deepening memory hierarchies to achieve lower Average Memory Access Time (AMAT) compared to UMA systems. NUMAs offer lower AMATs as combining memory requests satisfied by "local" memory (close to the processor with relatively low latency/energy) with "remote" accesses (with significantly higher latency/energy) results in lower AMAT compared to UMA systems which have constant latencies across different locations in memory. The ratio of local and remote memory accesses highly depends on the problem size and as data sets keep growing, NUMAs should increasingly provide larger local memory capacity to achieve high performance.

Caches already occupy up to $40 \%$ of the chip area [55] which not only significantly contributes to power consumption but also adds complexities and inefficiencies to the fabrication process due to the different technological requirements of SRAM cells compared to the compute logic. Moreover, NUMAs also impact the programmability of systems by further emphasizing the importance of data locality to achieve higher performance [23].

UMA designs, on the other hand, are easier to program but offer poor scalability as their AMAT is proportional to their size. A UMA with low average access latency could match the performance benefits of locality while providing the programming flexibility to fully exploit the compute power of the system independent from the problem size. Unfortunately, NUMAs emerged because UMAs have become prohibitively expensive for current system scales due to the energy and latency overheads of electrical interconnects.

S-UMA solves the NUMA challenge by utilizing a point-to-point $\mathrm{SiPh}$ fabric that enables uniform memory access without increasing
AMAT compared to NUMA by enabling low-latency access to both local and remote memories. Moreover, SiPhs enable UMA with large bisection bandwidth without excessive energy overheads even for large physical distances, offering significantly better scalability than electrical interconnects and could thereby enable scalable chiplet-based UMA systems.

\subsection{Addressing Disintegration Limits}

In addition to exploiting SiPhs to implement a UMA system with a scalable low-latency high-bandwidth interconnection network, we hypothesize that the scalability of SiPhs can also be leveraged to fuel further dis- and re-integration of processor and memory chiplets. In particular, we propose to disintegrate the L3 (LLC) cache, directory coherence, and memory controllers from the processor chiplet and re-integrate them into a separate chiplet which we term LLChiplet. In this scenario, as depicted in Figure 3, S-UMA consists of the following compute and memory chiplets in the same package:

Processor chiplets accommodate multiple cores with their corresponding L1I/L1D and L2 caches along with IO circuitry for interchiplet communication.

LLChiplets contain the LLC, the directory coherence controller, and the memory controller (MC). We consider an MC with a DDR4 DRAM interface in this paper, which could, however, be replaced by any other memory interface (e.g. serial HMC, HBM, etc.).

Co-locating the directory and memory controllers with the LLC on the same chiplet adds no overheads to the off-chip memory access time compared to designs monolithically integrating the controllers and LLC on the processor chiplet. Moreover, re-integration of L3s on the LLC chiplets eliminates the need for memory buffers (i.e., L4) by providing the same functionality through a flattened hierarchy-by bringing the LLCs and memory "closer" to each other.

With LLC sizes growing (currently up to $16 \mathrm{MiB}$ per chiplet [55]), enabling separate manufacturing processes for processor/memory chiplets allows the utilization of the most cost efficient process nodes and/or most power efficient for SRAM memory (e.g. by reducing leakage power of memory cells). Alternatively, non-volatile memory technologies like STT-RAM, which represent a promising solution to replace SRAM based LLCs [37], would also benefit from separate manufacturing as it reduces complexity of monolithic integration of different memory and processing technologies. 


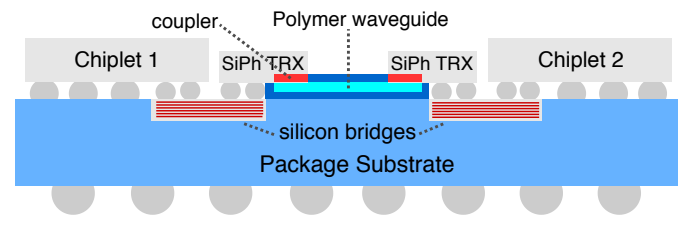

Figure 6: Optically-interconnected $\mathrm{SiPh}$ transceivers with transceiver-chiplet Si bridges on an organic substrate

Aside from these benefits, such disintegration of LLCs would increase access latency and energy as the LLC is no longer on the same chiplet and possibly far away on the package substrate; however, we hypothesize that our low-latency SiPh point-to-point fabric can mitigate these overheads to an extent where they do not cause noticeable performance degradation or energy overheads.

\subsection{Addressing the Packaging Challenge}

Figure 6 illustrates the cross-sectional view of our proposed packaging approach which loosely adopts a previous technique for interpackage communication [11] and applies it to inter-chiplet communication. Rather than monolithically integrating $\mathrm{SiPh}$ transceivers (TRXs) into processor chiplets, we propose to implement dedicated $\mathrm{SiPh}$ transceiver chiplets connected to their respective processor chiplet on one side through Si bridges and to each other through $\mathrm{SiPh}$ transceivers and polymer waveguides on the other side.

Polymer waveguides (PWGs) are integrated on top of the organic package substrate to provide optical connectivity between the chiplets. Scalable integration of PWGs (186 optical IOs [14]) has successfuly been demonstrated enabling high flexibility and connectivity in the interconnection network. Light is guided in and out of the chiplets using a vertical adiabatic coupler [14], which is a tapered waveguide (i.e., the waveguide width incrementally decreases) inside the chiplet placed on top of the on-package PWG. The tapering region of the waveguide confines the light and guides it into (and out of) the chiplets into/out of the PWG at low loss and low susceptibility to misalignment and mismatches. We refer the reader to the work of Dangel et al. $[13,14]$ for more details on the integration process.

Our approach combines SiPhs and Si bridges and utilizes each interconnection technology where it is the most efficient: SiPhs for long-distance interconnect between chiplets and Si bridges for short-distance interconnect between the TRXs and the chiplets. This approach does not reduce IO bandwidth density compared to direct optical communication between chiplets with monolithically integrated transceivers as the very fine-pitch electrical interconnects of Si bridges provide IO density similar to on-chip wires. Moreover, energy and latency overheads are negligible due to the small size of Si bridges and their low-loss vertical contacts [30].

Note that, while an additional TRX chip increases the distance between the actual chiplets compared to alternative techniques, the size of the TRX is very small as it only integrates transceiver circuitry and Si bridges enable tight packaging of just $100 \mu \mathrm{m}$ between the TRX and chiplets. The largest element on the TRX chip is the vertical adiabatic coupler, which, although providing very compact coupling width $(5-20 \mu \mathrm{m}[13,14])$ should be at least $200 \mu \mathrm{m}$ long to offer low-loss coupling (coupling loss decreases with longer coupling structures). Modeling with DSENT [61] on a 45nm technology node shows less than $1 \mathrm{~mm}^{2}$ for a transceiver matching the bandwidth of AMD's IF [3]. Nevertheless, these distance and area overheads will largely be outweighed by the benefits of this approach, which are

- significantly higher manufacturing yield compared to monolithic integration of SiPh TRX within the chiplets.

- more flexibility in choosing the most appropriate and efficient technology node and process to manufacture SiPh TRX and processor chiplets.

- elimination of any area concerns of $\mu \mathrm{m}$-scale SiPh devices compared to nm-scale CMOS.

While materials that are not silicon (e.g. Germanium) required in $\mathrm{SiPh}$ device manufacturing necessitate modifications to standard CMOS processes and therefore cannot exploit their infrastructure, $\mathrm{SiPh}$ manufacturing and photonic integrated circuit design (along with advanced tooling to increase productivity) have seen significant growth and investment in the last ten years, now allowing low-cost $\mathrm{SiPh}$ integration [65]. For a more detailed cost roadmap, we refer the reader to [32].

\section{METHODOLOGY}

System Comparisons. We compare S-UMA to representative stateof-the-art chiplet-based systems, the logical topologies of which are shown in Figure 7 (examples are with 4 processor chiplets for illustration purposes, all networks will be evaluated with 8 processor chiplets like in Figure 3). In particular, we compare a NUMA/NUCA system (similar to AMD's Naples [71]) shown in Figure 7a and a UMA/NUCA system (similar to AMD's Rome with a central electrical crossbar switch ("IO die") [55]) shown in Figure 7b to S-UMA both with (S-UMA-Dis) and without (S-UMA) LLC disintegration (Figure $7 \mathrm{~d}$ and $7 \mathrm{c}$, respectively) to independently study both the impact of a point-to-point optical fabric on current chiplet-based systems in general as well as the performance impact of LLC disintegration. Finally, we also add an electrical version S-UMA-Dis (S-UMA-E) to our study providing point-to-point interconnection with electrical links (unrealistic, infeasible in terms of cost due to IO pin limitations) to analyze the benefits of a technology shift towards optical interconnects.

Performance modeling. We used the cycle-level simulator gem5 [7] in full-system mode running the Linux operating system. For each network, we match the bandwidth of AMD's Infinity Fabric (IF) on the network links which provides $20 \mathrm{~GB} / \mathrm{s}(160 \mathrm{~Gb} / \mathrm{s})$ unidirectional link bandwidth ${ }^{2}$ and assume an electrical link traversal latency of 10ns [71], optical link traversal of 1ns [25, 68], 1 core clock cycle router traversal latency on the chiplets [71], and $25 \mathrm{~ns}$ latency through the IO die in Rome-like UMA-NUCA design. Table 2 lists the configurations of the systems under investigation.

We evaluate the networks with both synthetic and application traffic to study how the networks perform under a set of modern application workloads and how they would perform based on the network injection rate (which allows infering how other applications or multi-programmed workloads with much higher traffic

${ }^{2}$ IF operates at MEMCLK of DRAM $(2666 \mathrm{MHz}$ in our case) and has SERDES circuitry to transmit $4 \times 32-$ bits per MEMCLK [71] 


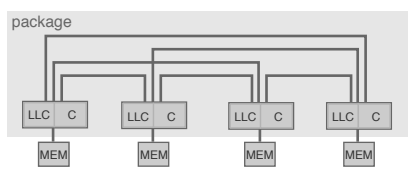

(a) Naples: Example of a NUMA system with NUCA

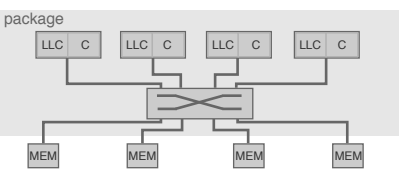

(b) Rome: Example of a NUCA system with UMA

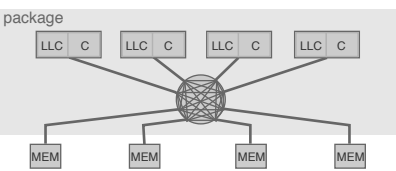

(c) S-UMA: System with UMA and NUCA

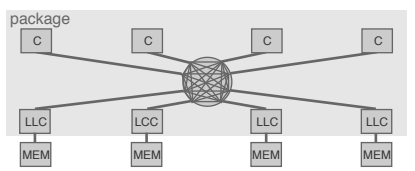

(d) S-UMA-Dis: Disintegrated SUMA with UMA and UCA

Figure 7: Logical topologies of chiplet-based systems with different memory architectures

Table 2: System configurations

\begin{tabular}{|c|c|c|c|}
\hline & Naples & Rome & S-UMA(-dis/-E) \\
\hline Topology & Hyper Cube & Star with Xbar & Point-to-point \\
\hline Config & \multicolumn{3}{|c|}{$\begin{array}{l}64 \text { x86 cores (8 chiplets) @ } 3 \mathrm{GHz} \\
\text { L1_I: } 64 \mathrm{~KB} / \text { core, assoc: } 4 \text {, private } \\
\text { L1_D: } 32 \mathrm{~KB} / \text { core, assoc: } 8 \text {, private } \\
\text { L2: } 512 \mathrm{~KB} / \text { core, assoc: } 8 \text {, private } \\
\text { L3: } 8 \mathrm{MB} /(8 \text { cores), assoc: } 16 \text {, shared } \\
\text { Memory: } 8 \text { Channels, DDR4, } 2 \mathrm{~GB}\end{array}$} \\
\hline
\end{tabular}

would perform). In synthetic workloads, packets consist of 4 flits with a flit width of 32 bits (according to AMD's IF which has a 32-bit wide interface) and apply uniform random and bit complement traffic to stress different corner cases of the topologies. For application workloads, we evaluate the chiplet-based systems for a variety of high-performance computing workloads from the NAS Parallel Benchmarks (NPB) with "C" server-class input sets [2], Rodinia [8], PARSEC3.0 and Splash-2x with large input sets [74], collecting statistics during the parallel region of the workloads. We chose various workloads with different data sharing and memory access patterns as well as working sets to identify the impact of S-UMA for a variety of application workloads.

Power modeling. We model the power consumption of the electrical interconnection fabrics based on the energy-per-bit values reported for AMD's IF in their chiplet-based systems, i.e., 2pJ/bit per [71] $]^{3}$. We used DSENT [61] for energy modeling of the switch and silicon bridge traversals based on a $14 \mathrm{~nm}$ technology node (modeling links on Si bridges as BEOL links).

We model power consumption of the $\mathrm{SiPh}$ links based on a demonstrated $25 \mathrm{~Gb} / \mathrm{s}$ transceiver from Li et al. $[39,73]$ in $65 \mathrm{~nm}$ CMOS (including static external laser power, serializer/deserializer, clock generation/recovery, drive rcircuitry, and microring tuning) combined with loss values corresponding to the SiPh packaging (i.e., demonstrated polymer waveguides and adiabatic couplers for both fiber-to-package and package-to-chiplet coupling [13, 14]) introduced in the previous section. In addition, we use SPICE models scaling down Li et al.'s transceiver to $28 \mathrm{~nm}$ and $14 \mathrm{~nm}$ to analyze power for both demonstrated technologies $(65 \mathrm{~nm} / 28 \mathrm{~nm}[9,39,51,73])$ and future projections (14nm). AWGR loss and crosstalk is based on a fabricated $16 \times 16 \mathrm{SiN}$ AWGR [54]. These assumptions based on manufactured and measured devices allows reporting realistic

${ }^{3}$ This is an optimistic assumption as 2pJ/bit in AMD's IF is consumed on intra-socket links. In Naples, some of the hypercube links are inter-socket links with higher pJ/bit. power consumption of the SiPh components. Tables 3 summarizes parameters and values.

Table 3: SiPh Device Parameters

\begin{tabular}{llll}
\hline Paramer & Value & Parameter & Value \\
\hline Optical Fiber & $5 \mathrm{e}-6 \mathrm{~dB} / \mathrm{cm}$ & Photodetector loss & $0.1 \mathrm{~dB}$ \\
Modulator Insertion loss & $1 \mathrm{~dB}$ & Power Margin & $3 \mathrm{~dB}$ \\
Waveguide loss & $0.5 \mathrm{~dB} / \mathrm{cm}$ & Filter through loss & $0.1 \mathrm{~dB}$ \\
Filter drop loss & $1.5 \mathrm{~dB}$ & AWGR loss & $1.8 \mathrm{~dB}$ \\
Coupler: Fiber-to-Package & $3 \mathrm{~dB}$ & AWGR crosstalk & $-20 \mathrm{~dB}$ \\
Coupler: Package-to-Chiplet & $0.5 \mathrm{~dB}$ & Laser efficiency & $14 \%$ \\
Receiver Sensitivity & $-17 \mathrm{dBm}$ & & \\
\hline
\end{tabular}

\section{SIMULATION RESULTS}

\subsection{Synthetic Workloads}

5.1.1 Performance. Figure 8 depicts the average packet latency results (note that S-UMA and S-UMA-Dis are based on the same network-only the type of nodes would change-which is why we left the latter out in these charts for clarity). The general trends are similar in both traffic patterns: as expected, S-UMA and S-UMA-Eboth based on all-to-all interconnection-offer both higher throughput due to higher bisection bandwidth and lower network latency due to lower average hop count compared to Naples (hypercube topology) and Rome (start/crossbar IO chip). In particular, network latency is significantly reduced by S-UMA as it not only has a lower diameter, but also lower link traversal latency due to optical communication (which is clearly more significant for inter-chiplet links than on-chip in NoCs where electrical communication over short distances can compete with optical links in terms of latency). Both latency critical applications and memory-bound workloads with high traffic between LLCs and/or off-chip memory would thus tremendously benefit from an optical all-to-all interconnect.

5.1.2 Power Consumption. Figure 9 shows the network power consumption for different injection rates. S-UMA-E consumes the highest power due to and the higher energy-per-bit compared to the SiPh links (2pJ/bit vs. $0.89 \mathrm{pJ} / \mathrm{bit}(14 \mathrm{~nm})$ vs. $1.124 \mathrm{pJ} / \mathrm{bit}(28 \mathrm{~nm})$ vs. $1.92 \mathrm{pJ} / \mathrm{bit}(65 \mathrm{~nm}))$ and the higher number of links compared to Naples and Rome (despite having a lower average number of hops). Although the electrical networks provide power consumption similar to S-UMA-E for low injection rates (mostly due to the static laser power overheads of the $\mathrm{SiPh}$ transceivers), S-UMA power efficiency becomes increasingly superior with rising injection rates due to its lower average hop count and lower-energy transceivers. S-UMA, therefore, not only provides higher bandwidth and lower latency, but also consumes less power, allowing system designers 
to dedicate more of the power budget towards the compute and memory-particularly for transceiver technologies below $65 \mathrm{~nm}$.

\subsection{Application Workloads}

5.2.1 Performance. Figure 10 illustrates the speed-up normalized to the Naples baseline. We observe that S-UMA provides an average speed-up of $23 \%$ compared to Naples and $12 \%$ compared to Rome, showing that the execution time of the considered workloads significantly benefits from our $\mathrm{SiPh}$ interconnection fabric. However, these speed-up benefits vary significantly. For instance, running Breadth First Search $(b f s)$ with S-UMA results in $2.3 \times$ speed-up compared to Naples as graph traversals significantly benefit from all-to-all connectivity. Workloads with smaller data sets and less frequent communications such as fft do not stress the interconnect as much, thus exhibiting only marginal speed-ups ( 10\%).

After analyzing the workloads, we discovered that the speed-up benefits mostly depend on 1) the amount of data sharing within an application, 2) (ir)regularities in memory access patterns, and 3) the working set size. Particularly 3) is an important attribute as our simulation set-up, like state-of-the-art chiplet-based systems, assumes very large on-chiplet cache $(1 \times 8 \mathrm{MiB} \mathrm{L} 3+8 \times 512 \mathrm{KiB})$, meaning that large parts of the working set fit into a chiplets cache, even when choosing the largest input sets available for such systems. In these scenarios, most of the traffic throughout workload execution is due to data sharing and coherence traffic (rather than cache capacity), leading to very low network utilization (and, in turn, over-provisioning of the networking resources). Nevertheless, as Figure 10 shows, we still see a fair speed-up of S-UMA compared to the electrical networks due to its much lower network latency.
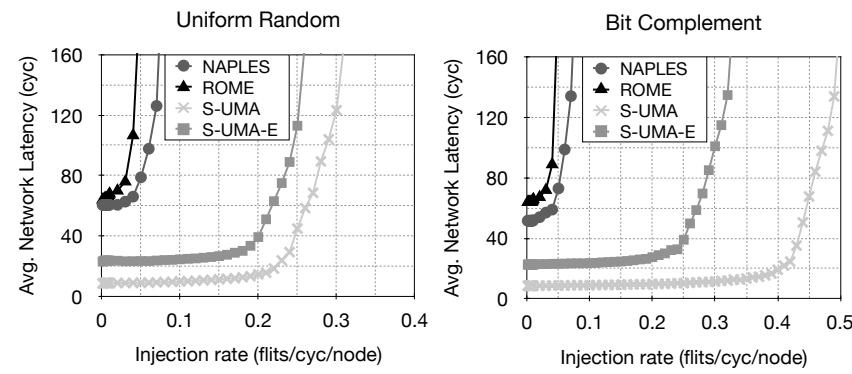

Figure 8: Average network latency (cycles) vs. injection rate (flits/cyc/node) under synthetic workloads
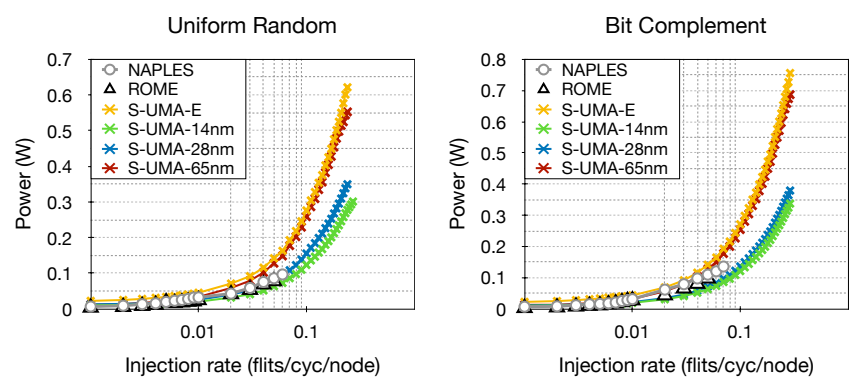

Figure 9: Power consumption (W) vs. injection rate (flits/cyc/node) under synthetic workloads
In the case of S-UMA-Dis, moving L3 caches off-chiplet increases L3 access latency compared to the other designs as on-chiplet accesses to a monolithically-integrated L3 are faster than inter-chiplet accesses; however, the speed and bandwidth provided by the all-toall optical interconnect minimizes these latency overheads for most applications. In fact, co-locating memory controllers and directories with L3 slices on a dedicated chiplet reduces inter-chiplet traffic in case of L3 misses due to cache conflicts since write-backs go straight to the memory and are not sent off-chiplet. For applications with relatively high L3 miss rates like bfs or radix (which exhibit almost random memory access patterns), this actually leads to a performance speed-up compared to S-UMA.

These results suggest that our $\mathrm{SiPh}$ point-to-point network allows to further exploit the benefits of chiplet disintegration while providing the benefits of a UMA design and still offering higher performance than state-of-the-art designs with less disintegration.

5.2.2 Power Consumption. Figure 11 shows the total power of each network normalized to Naples. On average, both S-UMA and S-UMA-Dis reduce power consumption by $30 \%$ compared to Naples and $48 \%$ compared to Rome for $14 \mathrm{~nm}$, which are both in line with our synthetic traffic results indicating that the lower average hop count and energy-per-bit of the SiPh TRXs offer significant power saving benefits. While our all-to-all topology with $28 \mathrm{~nm}$ TRXs matches power consumption of Naples on average, older $65 \mathrm{~nm}$ TRXs amount to significant power overheads. Generally, the higher the network utilization in the simulated workloads, the higher the power benefits of S-UMA(-Dis). This is mostly due to the fact that static power in $\mathrm{SiPh}$ (laser, MRR tuning) is a significant contributor to the total power and is amortized by high utilization.

\subsection{Discussion}

Our simulation results indicate that a point-to-point $\mathrm{SiPh}$ interconnection fabric can offer substantial improvements in terms of average network latency, application execution time, and power consumption compared to state-of-the-art electrical networks in chiplet-based systems . Not only does our proposal improve all figures of merit, it does so while enabling further disaggregation of LLC caches without performance hits and enables a scalable UMA architecture which will be significant to programmability and performance extraction of future systems. However, even with $\mathrm{SiPh}$, point-to-point networks will sooner or later reach its scalability limits. Although offering much better scalability than electrical networks and enabling architectures that would otherwise not be practical in the electrical domain,

The scalability of S-UMA will ultimately be limited by the crosstalk inside the AWGR, which limits its port count. Our analytical models of state-of-the-art SiN AWGRs [48, 54] show that they can scale up to $32 \times 32$ with acceptable power penalty, which would limit S-UMA to 32 chiplets for current AWGR technologies. However, AWGRs made from other materials like Silica scale to much higher port counts (up to $512 \times 512$ ), albeit with larger area footprint $\left(16 \mathrm{~mm} \times 11 \mathrm{~mm}=176 \mathrm{~mm}^{2}\right)$ [10]. Nevertheless, this area footprint might be negligible for S-UMA with +32 chiplets, which are $213 \mathrm{~mm}^{2}$ each in current designs [70].

Aside from scalability limits due to AWGR crosstalk, optical link bandwidth has a significant impact on laser power and MRR 


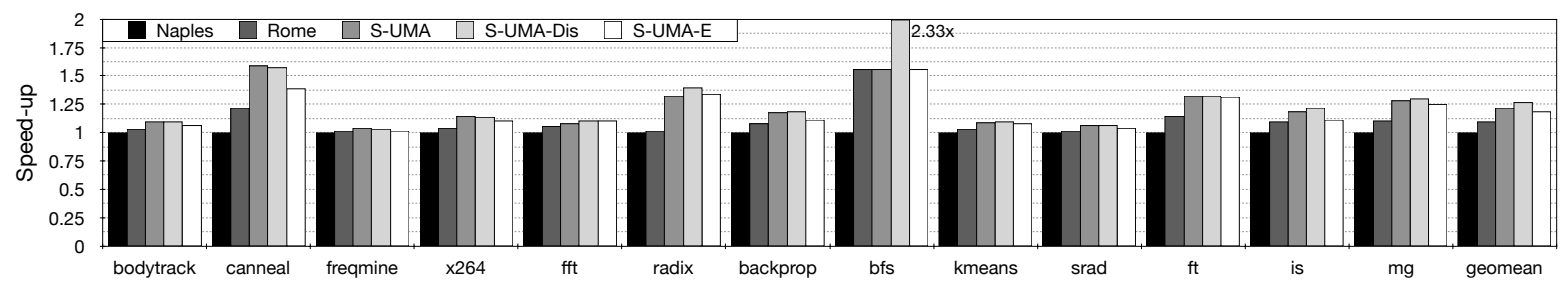

Figure 10: Speed-up normalized to Naples

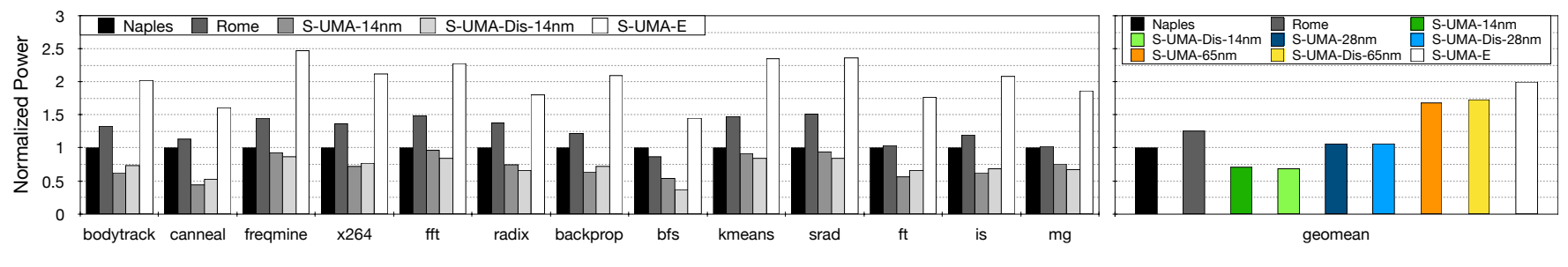

Figure 11: Power consumption normalized to Naples

tuning $[5,28,69])$, especially relative to network size. This might become a limiting factor to system scalability as the number of laser sources and their power impact the overall power efficiency of the system. Our results indicate, however, that for the workloads considered in our study, latency rather than bandwidth was the limiting factor. Therefore, for various HPC workloads considered in our study, link bandwidth could likely be reduced to save power and enable further scalability without significant performance hits.

Nevertheless, systems like multi-GPU systems have high link bandwidth demands, possibly making a straight-forward scaling of point-to-point networks power inefficient; however, as static laser power in $\mathrm{SiPh}$ is a well-known issue for such systems, several solutions such as adaptive laser sources which can be turned on and off based on the current bandwidth demands have been proposed to solve this issue [19-21, 49]. Chiplet-based systems in the HPC domain might particularly benefits from adaptive lasers as HPC systems have to process a variety of different workloads. Prior to launching applications, lasers could be turned on and off to provide the bandwidth necessary for the system. While we assume external off-chip/package lasers in our study, the time to control such lasers could be amortized by executing laser control in parallel to the setup phase of an application. Alternatively, on-chip lasers, although currently still suffering from low manufacturing yield and thermal issues, could be an attractive solution in the future as they allow nanosecond-scale control time, potentially even allowing dynamic bandwidth reconfiguration during an application execution.

Despite the scalability limits (which are still well-above what is possible in the electrical domain), the advancements and amount of research dedicated to $\mathrm{SiPh}$ devices is highly encouraging, with improvements in losses, laser efficiencies, and receiver sensitivities constantly published in the literature [63]. These have a significant impact on the total laser power could allow low laser power requirements even for high-bandwidth all-to-all fabrics in chiplet-based systems of medium or large scale, further fueling the scaling-up of shared memory chiplet-based HPC compute nodes and thereby reducing the data movement problem of scaling-out systems.

\section{CONCLUSION}

Given the increasing cost of monolithic chip design, multi-chip packages using smaller chiplets are becoming more common. However, these modular designs put significant strain on electrical interconnection networks leading to non-uniform memory architectures with large remote memory access latencies. In this paper, we showed that SiPhs can enable uniform memory architectures in chiplet-based systems with low memory access latency and energy. We exploit the high bandwidth density in optics to overcome issues arising due to IO pin bandwidth limitations and their wavelengthselective routing properties to enable point-to-point networks with low pin IO demands. Our results suggest that SiPhs could enable scalable chiplet-based uniform memory architectures and thus be of high importance to scale-up performance and, in turn, reduce the data movement overheads of scaling-out in HPC systems.

\section{ACKNOWLEDGMENT}

This work was supported in part by DoD contract \#H98230-16-C0820 and NSF grant \#1611560.

\section{REFERENCES}

[1] Akhil Arunkumar et al. 2017. MCM-GPU: Multi-chip-module GPUs for continued performance scalability. ACM SIGARCH Computer Architecture News 45, 2 (2017), $320-332$.

[2] David H Bailey. 2011. Nas parallel benchmarks. In Encyclopedia of Parallel Computing. Springer, 1254-1259.

[3] Noah Beck et al. 2018. 'Zeppelin': An SoC for multichip architectures. In ISSCC. IEEE.

[4] Stéphane Bellenger et al. 2014. Silicon interposers with integrated passive devices: Ultra-miniaturized solution using 2.5 D packaging platform. Caen, France, IPDiA White Paper Silicon Interposers_260214 (2014).

[5] Keren Bergman et al. 2014. Photonic network-on-chip design. Springer.

[6] Eric Beyne. 2016. The 3-D interconnect technology landscape. IEEE Design \& Test 33, 3 (2016), 8-20.

[7] Nathan Binkert, Bradford Beckmann, Gabriel Black, Steven K Reinhardt, Ali Saidi, Arkaprava Basu, Joel Hestness, Derek R Hower, Tushar Krishna, Somayeh Sardashti, et al. 2011. The gem5 simulator. ACM SIGARCH Computer Architecture News 39, 2 (2011), 1-7.

[8] Shuai Che et al. 2009. Rodinia: A benchmark suite for heterogeneous computing. In IISWC. Ieee, 44-54. 
[9] Yanfei Chen, Masaya Kibune, Asako Toda, Akinori Hayakawa, Tomoyuki Akiyama, Shigeaki Sekiguchi, Hiroji Ebe, Nobuhiro Imaizumi, Tomoyuki Akahoshi, Suguru Akiyama, et al. 2015. 22.2 A 25Gb/s hybrid integrated silicon photonic transceiver in 28nm CMOS and SOI. In 2015 IEEE International SolidState Circuits Conference-(ISSCC) Digest of Technical Papers. IEEE, 1-3.

[10] Stanley Cheung, Tiehui Su, Katsunari Okamoto, and SJB Yoo. 2014. Ultra-compact silicon photonic $512 \times 51225 \mathrm{GHz}$ arrayed waveguide grating router. IEEE fournal of Selected Topics in Quantum Electronics 20, 4 (2014), 310-316.

[11] Ian Cutress. [n. d.]. Intel launches Stratix-10-tx leveraging EMIB with 58G transceivers. https:/www.anandtech.com/show/12477/ intel-launches-stratix-10-tx-leveraging-emib-with-58g-transceivers-. [Online; accessed 11-14-2018].

[12] Ian Cutress. [n. d.]. Naples, Rome, Milan, Zen 4: An Interview with AMD CTO, Mark Papermaster. https://www.anandtech.com/show/13578/ naples-rome-milan-zen-4-an-interview-with-amd-cto-mark-papermaster. [Online; accessed 11-29-2018].

[13] Roger Dangel et al. 2015. Polymer waveguides for electro-optical integration in data centers and high-performance computers. Optics express 23, 4 (2015), 4736-4750.

[14] Roger Dangel et al. 2018. Polymer Waveguides Enabling Scalable Low-Loss Adiabatic Optical Coupling for Silicon Photonics. IEEE fournal of Selected Topics in Quantum Electronics 24, 4 (2018), 1-11.

[15] Sujal Das. 2018. It's Time for Disaggregated Silicon! https://www.netronome com/blog/its-time-disaggregated-silicon/. [Online; accessed 11-14-2018].

[16] Alexandre Ayres de Sousa. 2017. 3D Monolithic Integration: performance, Power and Area Evaluation for $14 n m$ and beyond. Ph.D. Dissertation. Université Grenoble Alpes.

[17] Behzad Dehlaghi and Anthony Chan Carusone. 2016. A 0.3 pJ/bit $20 \mathrm{~Gb} / \mathrm{s} /$ wire parallel interface for die-to-die communication. IEEE Journal of Solid-State Circuits 51, 11 (2016), 2690-2701.

[18] Yigit Demir et al. 2014. Galaxy: A high-performance energy-efficient multi-chip architecture using photonic interconnects. In ICS. ACM.

[19] Yigit Demir and Nikos Hardavellas. 2014. Ecolaser: an adaptive laser control for energy-efficient on-chip photonic interconnects. In 2014 IEEE/ACM International Symposium on Low Power Electronics and Design (ISLPED). IEEE, 3-8.

[20] Yigit Demir and Nikos Hardavellas. 2016. Energy-Proportional Photonic Interconnects. ACM Transactions on Architecture and Code Optimization (TACO) 13, 4 (2016), 54.

[21] Yigit Demir and Nikos Hardavellas. 2016. SLaC: Stage laser control for a flattened butterfly network. In 2016 IEEE International Symposium on High Performance Computer Architecture (HPCA). IEEE, 321-332.

[22] M. Doggett. [n. d.]. Xenos: XBOX360 GPU (2005). http://fileadmin.cs.lth.se/ cs/Personal/Michael_Doggett/talks/eg05-xenos-doggett.pdf. [Online; accessed 11-14-2018].

[23] Fabien Gaud, Baptiste Lepers, Jeremie Decouchant, Justin Funston, Alexandra Fedorova, and Vivien Quema. 2014. Large Pages May Be Harmful on NUMA Systems. In 2014 USENIX Annual Technical Conference (USENIX ATC 14). USENIX Association, Philadelphia, PA, 231-242. https://www.usenix.org/conference/ atc14/technical-sessions/presentation/gaud

[24] Michael Georgas, BR Moss, Chen Sun, Jeffrey Shainline, JS Orcutt, M Wade, Y-H Chen, Kareem Nammari, JC Leu, Aravind Srinivasan, et al. 2014. A monolithicallyintegrated optical transmitter and receiver in a zero-change $45 \mathrm{~nm}$ SOI process In VLSI Circuits Digest of Technical Papers, 2014 Symposium on. IEEE, 1-2.

[25] Paolo Grani et al. 2017. Design and Evaluation of AWGR-Based Photonic NoC Architectures for 2.5 D Integrated High Performance Computing Systems. In HPCA. IEEE.

[26] Paolo Grani, Gengchen Liu, Roberto Proietti, and SJ Ben Yoo. 2017. Bit-parallel all-to-all and flexible AWGR-based optical interconnects. In Optical Fiber Communication Conference. Optical Society of America, M3K-4.

[27] David Greenhill et al. 2017. A 14nm 1GHz FPGA with 2.5 D transceiver integration In ISSCC. IEEE, 54-55.

[28] Parisa Khadem Hamedani, Natalie Enright Jerger, and Shaahin Hessabi. 2014 Qut: A low-power optical network-on-chip. In NoCS. IEEE, 80-87.

[29] ibm. 2011. IBM zEnterprise 196 Technical Guide. (2011). https://www.redbooks. ibm.com/redbooks/pdfs/sg247833.pdf. [Online; accessed 11-14-2018].

[30] Intel. [n. d.]. Embedded Multi-Die Interconnect Bridge (EMIB). https://www.intel com/content/www/us/en/foundry/emib-an-interview-with-babak-sabi.html. [Online; accessed 11-14-2018]

[31] Subramanian S Iyer. 2018. Heterogeneous Integration using the Silicon Interconnect Fabric. In 2018 IEEE 2nd Electron Devices Technology and Manufacturing Conference (EDTM). IEEE, 135-137.

[32] Jeppix. [n. d.]. Cost Roadmap. http://www.jeppix.eu/new-page/. [Online; accessed 12-01-2018].

[33] Natalie Enright Jerger et al. 2014. Noc architectures for silicon interposer systems: Why pay for more wires when you can get them (from your interposer) for free? In MICRO. IEEE Computer Society, 458-470.
[34] Ajaykumar Kannan, Natalie Enright Jerger, and Gabriel H Loh. 2015. Enabling interposer-based disintegration of multi-core processors. In MICRO. IEEE, 546558.

[35] Ajaykumar Kannan, Natalie Enright Jerger, and Gabriel H Loh. 2016. Exploiting interposer technologies to disintegrate and reintegrate multicore processors. IEEE Micro 36, 3 (2016), 84-93.

[36] Pranay Koka, Michael O McCracken, Herb Schwetman, Xuezhe Zheng, Ron Ho, and Ashok V Krishnamoorthy. 2010. Silicon-photonic network architectures for scalable, power-efficient multi-chip systems. In ACM SIGARCH Computer Architecture News, Vol. 38. ACM, 117-128.

[37] Kunal Korgaonkar et al. 2018. Density tradeoffs of non-volatile memory as a replacement for SRAM based last level cache. In ISCA. IEEE.

[38] Cheng Li, Rui Bai, Ayman Shafik, Ehsan Zhian Tabasy, Geng Tang, Chao Ma, Chin-Hui Chen, Zhen Peng, Marco Fiorentino, Patrick Chiang, et al. 2013. A ring-resonator-based silicon photonics transceiver with bias-based wavelength stabilization and adaptive-power-sensitivity receiver. In Solid-State Circuits Conference Digest of Technical Papers (ISSCC), 2013 IEEE International. IEEE, 124-125.

[39] Hao Li et al. 2015. A $25 \mathrm{~Gb} / \mathrm{s}, 4.4 \mathrm{~V}$-swing, AC-coupled ring modulator-based WDM transmitter with wavelength stabilization in $65 \mathrm{~nm}$ CMOS. IEEE fournal of Solid-State Circuits 50, 12 (2015), 3145-3159.

[40] Gabriel H Loh et al. 2015. Interconnect-memory challenges for multi-chip, silicon interposer systems. In MEMSYS. ACM, 3-10.

[41] Joe Macri. 2015. AMD's next generation GPU and high bandwidth memory architecture: FURY. In HCS. IEEE, 1-26.

[42] Milo MK Martin, Mark D Hill, and Daniel J Sorin. 2012. Why on-chip cache coherence is here to stay. Commun. ACM 55, 7 (2012), 78-89.

[43] Sajjad Moazeni et al. 2017. A 40-Gb/s PAM-4 Transmitter Based on a RingResonator Optical DAC in 45-nm SOI CMOS. IEEE Journal of Solid-State Circuits 52, 12 (2017), 3503-3516.

[44] Nintendo. [n. d.]. Wii U announcement. http://iwataasks.nintendo.com/ interviews/\#/wiiu/console/0/0. [Online; accessed 11-19-2018].

[45] NVIDIA. 2017. NVIDIA Tesla V100 GPU Architecture. http://images.nvidia. $\mathrm{com} /$ content/volta-architecture/pdf/volta- architecture-whitepaper.pdf. [Online; accessed 03-14-2018].

[46] Deborah Patterson, Isabel De Sousa, and Lousi-Marie Achard. 2017. The future of packaging with silicon photonics. Chip Scale Review 21, 1 (2017).

[47] Stephen Phillips. 2014. M7: Next Generation SPARC. IEEE Hot Chips (2014).

[48] Molly Piels, Jared F Bauters, Michael L Davenport, Martijn JR Heck, and John E Bowers. 2014. Low-loss silicon nitride AWG demultiplexer heterogeneously integrated with hybrid III-V/silicon photodetectors. fournal of Lightwave Technology 32, 4 (2014), 817-823.

[49] Soumyajit Poddar, Prasun Ghosal, Priyajit Mukherjee, Suman Samui, and Hafizur Rahaman. 2012. Design of an NoC with on-chip photonic interconnects using adaptive CDMA links. In S2012 IEEE International OC Conference (SOCC). IEEE, 352-357.

[50] John W Poulton et al. 2013. A 0.54 pJ/b 20 Gb/s Ground-Referenced Single-Ended Short-Reach Serial Link in $28 \mathrm{~nm}$ CMOS for Advanced Packaging Applications. 7. Solid-State Circuits 48, 12 (2013), 3206-3218.

[51] Rajeev J Ram. 2015. Photonic-electronic integration with polysilicon photonics in bulk CMOS. In Silicon Photonics X, Vol. 9367. International Society for Optics and Photonics, $93670 \mathrm{~N}$.

[52] Suresh Ramalingam. 2014. 3D-ICs: Advances in the Industry. In Proc. IEEE 64th ECTC.

[53] Suresh Ramalingam. 2016. HBM package integration: Technology trends, challenges and applications. In Hot Chips 28 Symposium (HCS), 2016 IEEE. IEEE, $1-17$.

[54] Kuanping Shang et al. 2017. Low-loss compact silicon nitride arrayed waveguide gratings for photonic integrated circuits. IEEE Photonics fournal 9, 5 (2017), 1-5.

[55] Anton Shilov. 2018. AMD Previews Epyc 'Rome' Processor: Up to 64 Zen 2 Cores. https://www.anandtech.com/show/13561/ amd-previews-epyc-rome-processor-up-to-64-zen-2-cores. [Online; accessed 11-14-2018]

[56] Sergey Shumarayev. 2017. Stratix 10: Intel's 14nm Heterogeneous FPGA Systemin-Package (SiP) Platform. In HC29. IEEE.

[57] Balaram Sinharoy, JA Van Norstrand, Richard J Eickemeyer, Hung O Le, Jens Leenstra, Dung Q Nguyen, B Konigsburg, K Ward, MD Brown, José E Moreira, et al. 2015. IBM POWER8 processor core microarchitecture. IBM fournal of Research and Development 59, 1 (2015), 2-1.

[58] Phillip Stanley-Marbell et al. 2011. Pinned to the walls - Impact of packaging and application properties on the memory and power walls. In ISLPED. IEEE, 51-56.

[59] Dylan Stow et al. 2017. Cost-effective design of scalable high-performance systems using active and passive interposers. In ICCAD. IEEE Press, 728-735.

[60] Tiehui Su et al. 2018. Interferometric imaging using Si $3 \mathrm{~N} 4$ photonic integrated circuits for a SPIDER imager. Optics express 26, 10 (2018), 12801-12812.

[61] Chen Sun et al. 2012. DSENT-a tool connecting emerging photonics with electronics for opto-electronic networks-on-chip modeling. In NoCs. IEEE, 201-210.

[62] Chen Sun, Mark Wade, Michael Georgas, Sen Lin, Luca Alloatti, Benjamin Moss, Rajesh Kumar, Amir Atabaki, Fabio Pavanello, Rajeev Ram, et al. 2015. A 45nm 
SOI monolithic photonics chip-to-chip link with bit-statistics-based resonant microring thermal tuning. In 2015 Symposium on VLSI Circuits (VLSI Circuits) IEEE, C122-C123.

[63] David Thomson et al. 2016. Roadmap on silicon photonics. fournal of Optics 18 7 (2016), 073003.

[64] Ahmad Usman et al. 2017. Interposer Technologies for High-Performance Applications. IEEE Transactions on Components, Packaging and Manufacturing Technology 7, 6 (2017), 819-828.

[65] Jian Wang and Yun Long. 2018. On-chip silicon photonic signaling and processing: a review. Science Bulletin (2018).

[66] Sebastian Werner et al. 2018. AWGR-based Optical Processor-to-Memory Communication for Low-latency, Low-energy Vault Accesses. In MEMSYS. ACM.

[67] Sebastian Werner et al. 2018. Towards Energy-Efficient High-Throughput Photonic NoCs for 2.5 D Integrated Systems: A Case for AWGRs. In NOCS. IEEE.

[68] Sebastian Werner, Javier Navaridas, and Mikel Luján. 2015. Amon: An advanced mesh-like optical noc. In HOTI. IEEE, 52-59.
[69] Sebastian Werner, Javier Navaridas, and Mikel Luján. 2017. A Survey on Optical Network-on-Chip Architectures. ACM Computing Surveys (CSUR) 50, 6 (2017), 89.

[70] Wikichip.org. [n. d.]. EPYC 7601 - AMD. https://en.wikichip.org/wiki/amd/epyc/ 7601. [Online; accessed 04-01-2019].

[71] Wikichip.org. [n. d.]. Zen - Microarchitectures - AMD. https://en.wikichip.org/ wiki/amd/microarchitectures/zen\#Die-die_memory_latencies. [Online; accessed 11-19-2018].

[72] Jieming Yin et al. 2018. Modular Routing Design for Chiplet-based Systems. In ISCA. IEEE, 726-738.

[73] Kunzhi Yu et al. 2016. A $25 \mathrm{~Gb} / \mathrm{s}$ hybrid-integrated silicon photonic sourcesynchronous receiver with microring Wavelength Stabilization. IEEE fournal of Solid-State Circuits 51, 9 (2016), 2129-2141.

[74] Xusheng Zhan, Yungang Bao, Christian Bienia, and Kai Li. 2017. PARSEC3. 0: A multicore benchmark suite with network stacks and SPLASH-2X. ACM SIGARCH Computer Architecture News 44, 5 (2017), 1-16. 